\title{
Effect of Vildagliptin, Compared to Sitagliptin, on the Onset of Hyperglycemia-Induced Metabolic Memory in Human Umbilical Vein Endothelial Cells
}

\author{
La Sala $L^{1}$, Genovese $S^{2}$ and Ceriello $A^{1-3 *}$ \\ ${ }^{1}$ Department of Cardiovascular Research, IRCCS, Multimedica, Milan, Italy \\ ${ }^{2}$ Department of Cardiovascular and Metabolic Diseases, IRCCS, MultiMedica, Sesto San Giovanni, Milan, Italy \\ ${ }^{3}$ Biomedique Research Institute August Pi y Sunyer (IDIBAPS), Barcelona, Spain
}

\begin{abstract}
Background: Metabolic memory, the long-term effect of poor glycemic control in the initial stages of diabetes, leads to vascular complications that negatively affect patients' outcome. As oxidative stress plays a major role in metabolic memory onset, the use of drugs with antioxidant properties may be clinically beneficial.

Objectives: To test the effects of two dipeptidyl peptidase-4 inhibitors, vildagliptin and sitagliptin, on hyperglycemia-induced oxidative stress and apoptosis in Human Umbilical Vein Endothelial Cells (HUVECs).

Methods: HUVECs were treated with $5 \mathrm{nM}$ vildagliptin or sitagliptin for $1 \mathrm{~h}$, after 21 days of culture under conditions of continuous normal or high glucose (NG and HG, respectively), Oscillating Glucose (OG) or HG/OG memory ( $\mathrm{HM}$ and $\mathrm{OM}$, respectively). The effects of the two drugs on the following markers of oxidative stress were tested by different techniques: Reactive Oxygen Species (ROS), 8-hydroxy-deoxy-guanosine, 3-nitrotyrosine, thioredoxin-interacting protein (TXNIP) mRNA and PKC- $\beta$ protein. Moreover, the levels of BCL-2 (anti-apoptotic) and BAX (pro-apoptotic) transcripts and of caspase-3 protein were assayed.

Results: In HUVECs, vildagliptin was able to significantly counteract the oxidative stress triggered by OG, HG and memory conditions, as measured by the levels of ROS, DNA and protein damage markers, TXNIP and PKC- $\beta$. Also, a significant increase of BCL-2 and decrease of BAX mRNA levels was observed upon OG and HG. Sitagliptin exerted a less evident effect. No significant effect on caspase-3 levels was detected by either drug.

Conclusions: Our findings point toward antioxidant and antiapoptotic properties of vildagliptin in HUVECs exposed to HG, OG and metabolic memory conditions, whereas the effects of sitagliptin were less prominent. If these results on the vascular protective effects of vildagliptin will be confirmed, its use may be implemented in the setting of diabetes to prevent the onset of metabolic memory.
\end{abstract}

Keywords: Apoptosis; Endothelial cells; Hyperglycemia; Metabolic memory; Oxydative stress; Sitagliptin; Vildagliptin

\section{Introduction}

Type 2 Diabetes (T2D) is a syndrome characterized by hyperglycemia, which plays a major role in the pathogenesis of T2Dassociated complications like cardiovascular disease and nephropathy. Hyperglycemia induces oxidative stress [1-5], and increased levels of Reactive Oxygen Species (ROS) exert a detrimental effect on cellular functions by damaging lipid membranes, enzymes and nucleic acids, and by triggering apoptosis [6]. In addition to continuous hyperglycemia, post-prandial oscillations of glucose levels, like those experienced daily by diabetic patients, have been suggested as an independent risk factor for both microvascular and macrovascular complications [7-9]. This concept is consistent with the finding that intermittent High Glucose (HG) levels lead to increased damage to endothelial cells [10-13], mesangial cells [14] and tubule-interstitial cells in vitro [15]. Of note, it has been demonstrated that oscillating glucose levels result in increased levels of markers of ROS and vascular stress in both cultured endothelial cells and diabetic patients $[3,4,10$ 13,16]. It has also been shown that following a hyperglycemic stress, the levels of basement membrane proteins such as collagen and fibronectin, which are markers of high glucose stress, remain elevated several days after glucose levels have normalized [16,17]. This phenomenon, defined as "metabolic memory", has been linked to poor glycemic control in the early phases of diabetes, despite a tight glycemic control achieved during the course of the disease [18-20]. Several preclinical and clinical studies have demonstrated the existence of a metabolic memory in endothelial cells [19]. Moreover, it has been shown that normalization of the extracellular glucose level does not switch off the intracellular pro-oxidant environment, suggesting a role for ROS in the onset of cellular memory [16]. Of great interest is the observation that glucose fluctuations also produce a memory in Human Umbilical Vein Endothelial Cells (HUVECs), likely through the generation of oxidative stress [5]. Therefore, from a clinical point of view, agents able to counteract the phenomenon of metabolic memory in endothelial cells may help preventing vascular complications that negatively affect the outcome of diabetic patients.

In diabetes, hyperglycemia also impairs the protective action exerted by Glucagon-Like Peptide-1 (GLP-1) on endothelial function [21-22],

*Corresponding author: Antonio Ceriello, Department of Cardiovascular Research, IRCCS MultiMedica, Via Milanese, 300 Sesto San Giovanni (MI), Milan, Italy, Tel: +39 02 55406587; Fax: +39 02 55406503; E-mail: antonio.ceriello@multimedica.it

Received December 15, 2016; Accepted January 10, 2017; Published January 17,2017

Citation: Sala LL, Genovese S, Ceriello A (2017) Effect of Vildagliptin, Compared to Sitagliptin, on the Onset of Hyperglycemia-Induced Metabolic Memory in Human Umbilical Vein Endothelial Cells. Cardiovasc Pharm Open Access 6: 203. doi: 10.4172/2329-6607.1000203

Copyright: @ 2017 Sala LL, et al. This is an open-access article distributed under the terms of the Creative Commons Attribution License, which permits unrestricted use, distribution, and reproduction in any medium, provided the original author and source are credited. 
Citation: Sala LL, Genovese S, Ceriello A (2017) Effect of Vildagliptin, Compared to Sitagliptin, on the Onset of Hyperglycemia-Induced Metabolic Memory in Human Umbilical Vein Endothelial Cells. Cardiovasc Pharm Open Access 6: 203. doi: 10.4172/2329-6607.1000203

Page 2 of 6

which can be effectively restored by the use of Dipeptidyl Peptidase- 4 (DPP-4) inhibitors [23]. Among these, vildagliptin and sitagliptin have demonstrated antioxidant effects in both pre-clinical and clinical studies, significantly lowering oxidative stress-related markers [2430]. Moreover, a recent report has shown that treatment of HUVECs with the DDP-4 inhibitor teneligliptin improved the oxidative stress and apoptotic phenotype induced by exposure to continuous HG [31]. Notably, this agent could also overcome the detrimental effects triggered by HG-induced metabolic memory conditions.

As data on the effects of vildagliptin and sitagliptin on metabolic memory in endothelial cells are currently lacking, we tested whether these inhibitors could counteract, in HUVECs, hyperglycemia-induced oxidative stress damage and apoptosis and switch-off the metabolic memory caused by exposure to either oscillating or continuous HG levels.

\section{Materials and Methods}

\section{Cell culture and experimental design}

HUVECs were purchased from Lonza (Lonza Bioresearch LBS, Basel, Switzerland) and cultured with $\mathrm{EGM}^{\mathrm{mat}}-2$ Bulletkit ${ }^{\mathrm{m}}$ supplemented with human epidermal growth factor (hEGF), hydrocortisone, human recombinant fibroblast growth factor-beta (hFGF-b), heparin, $2 \%$ fetal bovine serum (FBS), and gentamicin/amphotericin-B (GA), at $37^{\circ} \mathrm{C}$ in a humidified atmosphere with $5 \% \mathrm{CO}_{2}$.

Cells were used at passage 4 and were plated in duplicate at $2 \times 10^{5}$ in 6-well plates for total RNA and protein extraction, and at $1 \times 10^{4}$ in 96-well plate for ROS measurement. After seeding, they were allowed to attach overnight and, after $24 \mathrm{~h}$, were exposed to one of the following glucose conditions:

1. Continuous normal glucose (NG) $(5 \mathrm{mM})$ for 21 days.

2. Oscillating glucose $(\mathrm{OG})(5-25 \mathrm{mM})$ for 21 days.

3. Continuous high glucose (HG) $(25 \mathrm{mM})$ for 21 days.

4. Cellular oscillating glucose memory $(\mathrm{OM})$ : OG (5-25 mM) for 14 days, followed by NG ( $5 \mathrm{mM}$ ) for 7 days.

5. Cellular High glucose memory (HM): continuous HG (25 mM) for 14 days, followed by NG ( $5 \mathrm{mM})$ for 7 days.

After 21 days, vildagliptin ( $5 \mathrm{nM})$ or sitagliptin $(5 \mathrm{nM})$ were added to the culture media for $1 \mathrm{~h}$.

\section{Measurement of ROS and of oxidative stress markers}

The fluorescent probe $2^{\prime}, 7^{\prime}$-Dichlorofluorescein diacetate ( $\left.\mathrm{H}_{2} \mathrm{DCFDA}\right)$ was used to measure the intracellular generation of ROS. $1 \times 10^{4}$ HUVECs were grown in clear flat bottom treated 96-well plates during 21 days. At the end of this period, cells were treated with the above-mentioned drugs, and after removing the medium, they were stained with $20 \mu \mathrm{M} \mathrm{H}_{2}$ DCFDA for $30 \mathrm{~min}$ at $37^{\circ} \mathrm{C}$. The fluorescence intensity of $\mathrm{H}_{2}$ DCFDA was measured kinetically at an excitation and emission wavelength of $485 \mathrm{~nm}$ and $530 \mathrm{~nm}$, respectively, for $\mathrm{H}_{2}$ DCFDA using a fluorescent microplate reader (Synergy HT, BioTek Instruments, Inc., Winooski, Vermont, USA).

The content of 8-hydroxy-deoxy-guanosine (8-OH-dG, StressMarq biosciences, Victoria, Canada), and 3-nitrotyrosine (3-NY) was measured by ELISA (Abcam, Cambridge, USA), following the manufacturer's instructions.

\section{RNA isolation and $\mathrm{qRT}-\mathrm{PCR}$}

Total RNA was isolated from HUVECs using the total RNA isolation kit (NorgenBiotek Corp, Thorold, Ontario, Canada) following the manufacturer's instructions. The first-strand cDNA was prepared using 1-2 $\mu$ g of total RNA, the Superscript III RT kit and the random hexamer primers (Invitrogen, Carlsbad, CA, USA) in a total volume of $25 \mu \mathrm{l}$ according to the manufacturer's instructions. Quantitative realtime PCR (qRT-PCR) was performed on a QuantStudio 6 flex (Applied biosystem) detection system using the SybrGreen reagents (Takara Bio Company, Clontech, Mountain View, CA, USA) and TaqMan Gene Expression Master Mix (Applied Biosystems, Foster City, CA, USA). Primers used are listed in Table 1.

\section{Protein analysis}

For western blot analysis, HUVECs were lysed in RIPA buffer (Sigma-Aldrich) with $10 \%$ protease and $1 \%$ phosphatase inhibitors (Sigma-Aldrich). After determining the content of protein by the Bradford assay buffer (Sigma-Aldrich), $50 \mu \mathrm{g}$ of lysates were separated by electrophoresis using PAGEr gels (4-12\%) (Lonza) and transferred onto a PVDF membrane (Perkin Elmer, Waltham, MA, USA). After blocking with $5 \%$ non-fat dried milk or $5 \%$ bovine serum albumin, membranes were incubated with the respective primary antibodies (1:1,000 anti-PKC- $\beta$ [Santa Cruz, Santa Cruz, CA, USA], 1:500 anticaspase- 3 [Abcam, Cambridge, UK] and 1:1,000 anti- $\beta$-actin [SigmaAldrich, St Louis, MO, USA) overnight at $4^{\circ} \mathrm{C}$. Membranes were then incubated with the appropriate secondary horseradish peroxidaseconjugated IgG antibodies (GE Healthcare Europe GmbH, Milan, Italy) at a 1:3,000 dilution for $1 \mathrm{~h}$ at room temperature. Blots were revealed by LI-COR ECL Reagent and C-DiGit Blot scanner (LI-COR Biosciences). Protein spots were quantitated by Image Studio software (www.licor.com). $\beta$-actin served as the loading control.

\section{Statistical analysis}

All experiments were performed eight independent times. Numerical data were expressed as mean \pm standard error of the mean (SEM). Statistical analysis was performed using the one-way analysis of variance (ANOVA) with appropriate post-hoc multi-comparison tests (Tukey's), to compare the effects of vildagliptin and sitagliptin. A P value of $<0.05$ was considered statistically significant.

\section{Results}

\section{Effects of DDP-4 inhibitors on oxidative stress and damage}

ROS content was measured by a fluorometric assay under 5 different glucose conditions. In untreated cells, exposure to HG (oscillating [OG], continuous [HG], or mimicking cellular memory under oscillating $[\mathrm{OM}]$ or continuous $[\mathrm{HM}])$, but not to $\mathrm{NG}$, significantly increased the production of ROS, and did so at a similar extent. Compared to untreated cells, treatment with vildagliptin significantly decreased

\begin{tabular}{|c|c|}
\hline Gene & $\begin{array}{c}\text { Sequence } \\
\text { TXNIP }\end{array}$ \\
\hline$\beta-A C T I N$ & $\begin{array}{c}\text { GCAGTGCAAACAGACTTCGG } \\
\text { TCACCTGTTGGCTGGTCTTC }\end{array}$ \\
\hline BAX & $\begin{array}{c}\text { CAGCCATGTACGTTGCTATCCAGG } \\
\text { AGGTCAGACGCAGGATGGCATG }\end{array}$ \\
\hline BCL-2 & TCAGGATGCGTCCACCAAGAAG \\
& TGTGTCCACGGCGGCAATCATC \\
\hline ATCGCCCTGTGGATGACTGAGT \\
GCCAGGAAATCAAACAGAGGC
\end{tabular}

Table 1: Primers used to assess the transcript levels of the following genes by SYBR-green quantitative-Real Time-PCR, in HUVECs either untreated or treated with vildagliptin or sitagliptin upon different glucose conditions. 
the content of ROS in cells grown under cellular memory conditions (OM: $\mathrm{p}<0.05$; HM: $\mathrm{p}<0.01$; Figure $1 \mathrm{~A}$ ). Notably, these cells displayed a significantly lower content of ROS compared to cells grown under OG and HG, respectively. No significant difference was observed upon exposure to sitagliptin under any glucose conditions.

Next, we evaluated the levels of $8-\mathrm{OH}-\mathrm{dG}$ and $3-\mathrm{NY}$, which are markers of hyperglycemia-induced DNA and protein damage. As shown in Figures $1 \mathrm{~B}$ and $1 \mathrm{C}$, in untreated cells, exposure to any condition but HM significantly increased the levels of these markers compared to NG. Treatment with vildagliptin but not sitagliptin was able to counteract both markers in cells grown under HM conditions $(\mathrm{p}<0.05)$.

\section{Effects of DDP-4 inhibitors on TXNIP transcript levels}

We then assessed the transcript levels of thioredoxin-interacting protein (TXNIP). In untreated cells, compared to NG, all HG conditions triggered a significant up-regulation of TXNIP mRNA (Figure 2). Vildagliptin-treated cells grown under $\mathrm{OM}$ and $\mathrm{HM}$ conditions displayed a significant decrease of transcript compared to untreated cells ( $\mathrm{p}<0.01$ in both cases), which was similar to that observed in cells grown under NG. Conversely, cells grown under OG and HG had higher levels than those upon NG. The effect was less marked after sitagliptin treatment.

\section{Effects of DDP-4 inhibitors on PKC- $\beta$ mRNA levels}

Prolonged exposure of HUVECs to OG, HG and memory conditions induced an increase in PKC- $\beta$ levels, as demonstrated by Western blot (Figure 3). Following treatment with vildagliptin and sitagliptin, the levels of this marker decreased, although differences were not statistically significant. However, under OM and HM conditions, the expression was significantly lower than under OG and HG, respectively.

\section{Effects of DDP-4 inhibitors on apoptosis-related markers' levels}

The mRNA levels of the apoptosis-related markers BCL-2 (antiapoptotic) and BAX (pro-apoptotic) were evaluated by qPCR. In untreated cells, compared to NG conditions, exposure to any of the 4 HG conditions did not alter the levels of BCL-2 mRNA (Figure 4A), while it significantly increased those of BAX (Figure 4B). Treatment with vildagliptin increased BCL-2 transcript levels, but, compared to untreated cells grown in NG, the difference was significant only upon $\mathrm{HM}(\mathrm{p}<0.05$, Figure $4 \mathrm{~A})$. No difference was observed following treatment with sitagliptin (Figure 4A). In contrast, levels of BAX mRNA decreased following treatment with both drugs, but the difference was significant only in cells grown under OM $(\mathrm{p}<0.01)$ and HM $(\mathrm{p}<0.05)$ and exposed to vildagliptin, and in those grown under OM $(\mathrm{p}<0.01)$ and exposed to sitagliptin (Figure 4B).
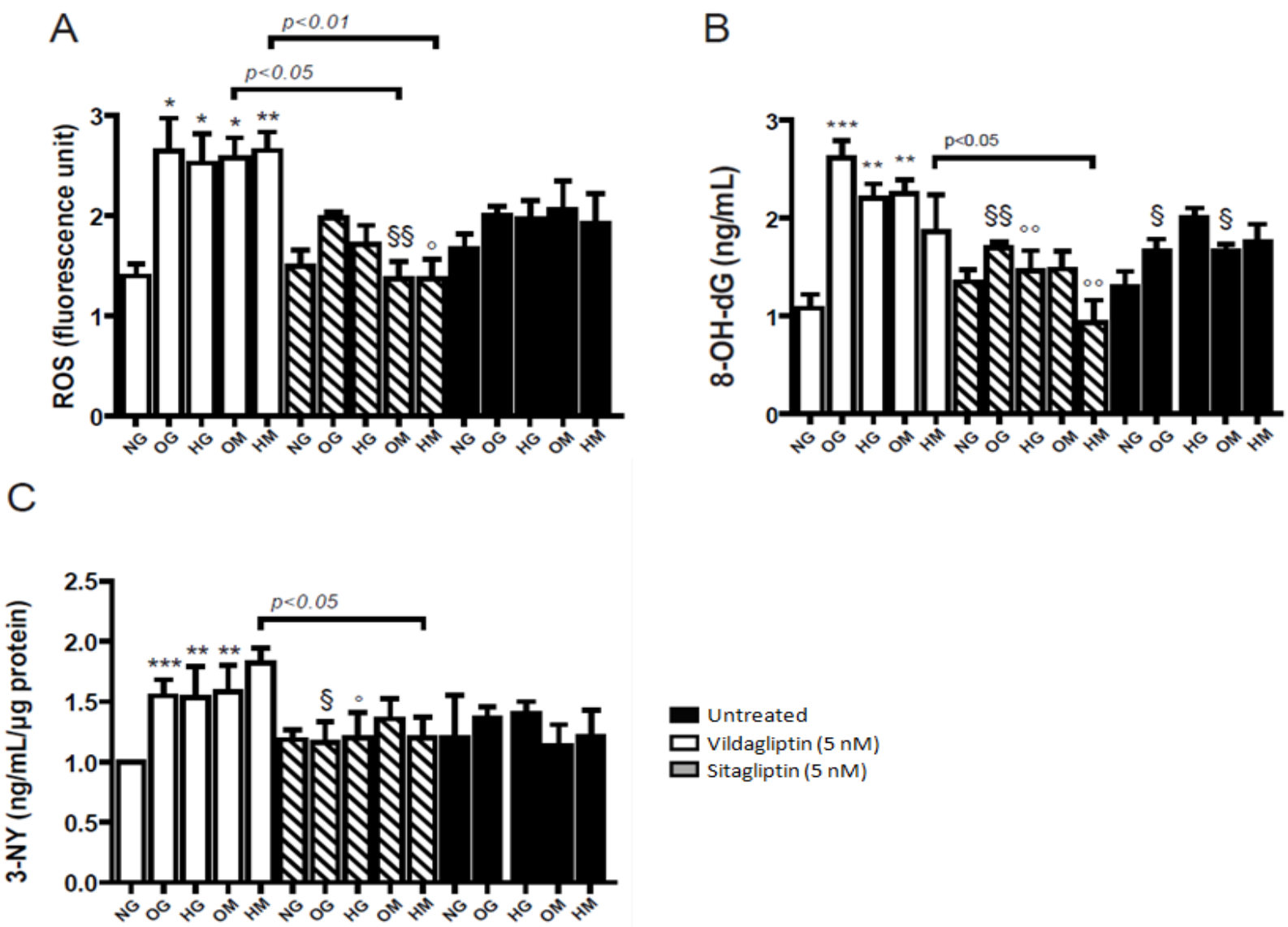

Figure 1: Oxidative stress markers' assessment. (A) Total ROS intensity measurement was performed in HUVECs after 21 days in culture under conditions of NG, $\mathrm{OG}, \mathrm{HG}$ or OM-HM. Cells were treated with vildagliptin $(5 \mathrm{nM})$ or sitagliptin $(5 \mathrm{nM})$ for $1 \mathrm{~h}$. (B) $8-\mathrm{OH}-\mathrm{dG}$ content was evaluated under the aforementioned conditions by ELISA. (C) 3-NY content was measured by ELISA and normalized to total protein content. Bars represent mean \pm SEM. One-way ANOVA, followed by Tukey's posthoc test was performed. Symbols represent the result of the comparison with untreated cells under $\mathrm{NG}^{*}, \mathrm{OG}^{\S}$ and $\mathrm{HG}^{\circ}$. " $\mathrm{p}<0.05,{ }^{* *} \mathrm{p}<0.01,{ }^{* * *} \mathrm{p}<0.001,{ }^{\S} \mathrm{p}<0.05,{ }^{\$ \S} \mathrm{p}<0.01$, " $\mathrm{p}<0.05,{ }^{\circ} \mathrm{p}<0.01$. ROS: reactive oxygen species; NG: normal glucose; OG: oscillating high glucose; HG: continuous high glucose; OM: OG memory; HM: HG memory; 8-OH-DG: 8-hydroxy-deoxy-guanosine; 3-NY: 3-nitrotyrosine. 


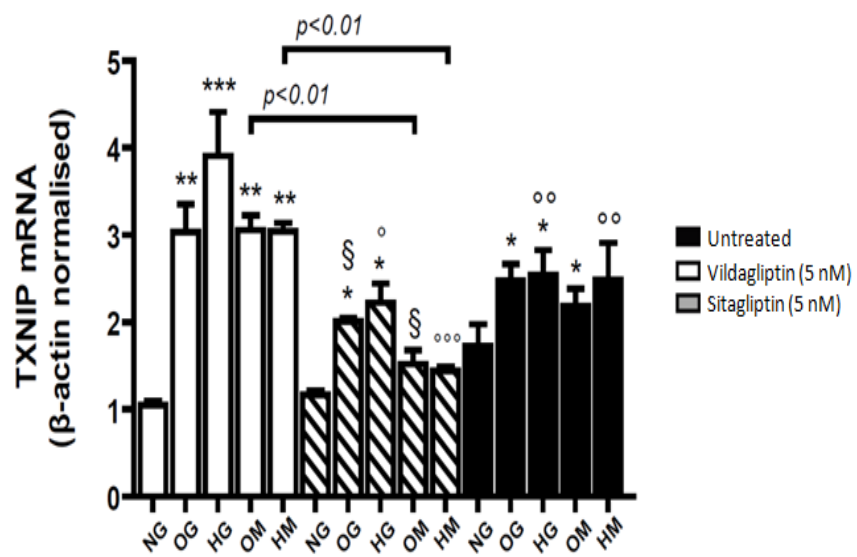

Figure 2: Expression of the oxidative stress marker TXNIP mRNA. Total cellular RNA was isolated from HUVECs after 21 days in culture under NG, OG, HG or $\mathrm{OM}-\mathrm{HM}$ conditions. Cells were treated with vildagliptin $(5 \mathrm{nM})$ or sitagliptin $(5$ $\mathrm{nM}$ ) for $1 \mathrm{~h}$. The levels of mRNA encoding for TXNIP were assessed by qRTPCR and normalized on $\beta$-actin. The experiment was performed three times. Bars represent mean \pm SEM. One-way ANOVA, followed by Tukey's post-hoc test was performed, where * vs. NG, $\S$ vs. OG, and ${ }^{\circ}$ vs. HG. ${ }^{*} p<0.05,{ }^{* *} p<0.01,{ }^{* * *} p<0.001$, $\$ p<0.05, " p<0.05,{ }^{\circ} p<0.01,{ }^{\circ} p<0.001$. TXNIP: Thioredoxin-interacting protein; NG: Normal glucose; OG: Oscillating high glucose; HG: Continuous high glucose; OM: OG memory; HM: HG memory.

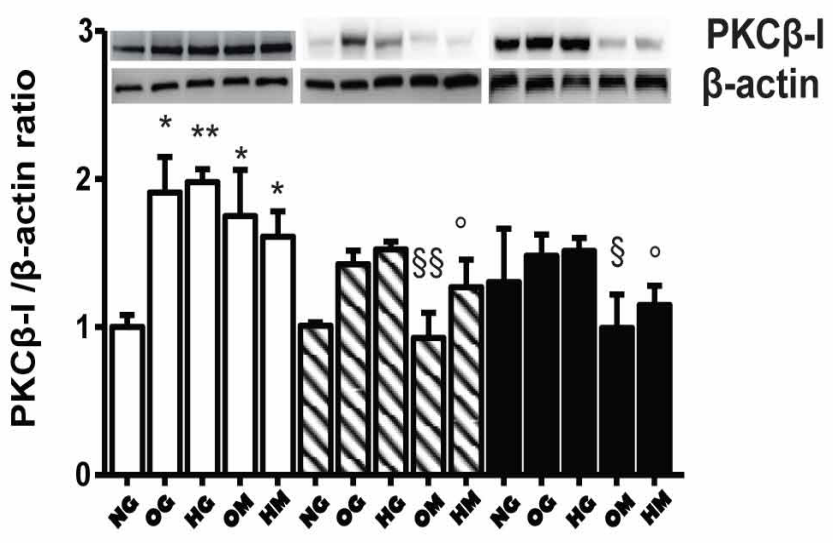

Figure 3: PKC- $\beta$ expression. Total whole cell lysates were prepared from HUVECs grown under different glucose conditions per 21 days and then treated with vildagliptin $(5 \mathrm{nM})$ or sitagliptin $(5 \mathrm{nM})$ for $1 \mathrm{~h} .50 \mu \mathrm{g}$ of lysates were resolved on SDS-PAGE and incubated with an anti-PKC- $\beta$ antibody. The levels of protein were normalized on $\beta$-actin. The experiment was performed three times. Bars represent mean \pm SEM. One-way ANOVA, followed by Tukey's post-hoc test was performed, where * vs. NG, ${ }^{\star}$ vs. OG, and ${ }^{\circ}$ vs. HG. ${ }^{*} p<0.05,{ }^{*} p<0.01,{ }^{\S} p<0.05$, $\$ \S p<0.01, \quad p<0.05$. NG: Normal glucose; OG: Oscillating high glucose; HG: Continuous high glucose; OM: OG memory; HM: HG memory.

Finally, we assessed the levels of caspase-3, a marker of apoptotic cell death. In untreated cells, a significant increase was observed only under HG vs NG. Treatment with vildagliptin led to a significant decrease only in cells exposed to HM $(\mathrm{p}<0.05)$, whereas no effect of sitagliptin was observed.

\section{Discussion and Conclusion}

Compelling evidence has demonstrated that metabolic memory is caused by poorly controlled hyperglycemia in the early stages of diabetes, and leads to vascular complications regardless of tight glucose control attained during the course of disease. Occurrence of this phenomenon has been reported in endothelial cells, in which it is driven by different mechanisms, such as oxidative stress and epigenetic changes [19]. DDP-4 inhibitors are anti-diabetic agents with vascular protective effects [32]. Yet, little is known about their impact on HG-induced stress and metabolic memory in endothelial cells. To the best of our knowledge, this is the first study addressing the effects of vildagliptin and sitagliptin on the levels of different markers of oxidative stress and apoptosis in HUVECs grown under conditions of both oscillating and continuous HG and HG-induced metabolic memory. The levels of several well established markers of oxidative stress were measured: ROS, which are important mediators of hyperglycemia-induced tissue damage [33] 8-OH-dG, a marker of oxidative DNA damage and of macrovascular complications in T2D patients [34], 3-NY a marker of ROS-induced protein damage [16], TXNIP, whose levels increase upon ROS production and hyperglycemia [35] and PKC- $\beta$ [36], a mediator of hyperglycemia-induced vascular effects. Moreover, we selected BCL2 , BAX and caspase- 3 as markers of apoptosis.

In untreated cells, compared to NG conditions, exposure to HG increased the levels of oxidative stress markers and of the apoptotic markers BAX and caspase-3, while leaving the levels of BCL-2 unchanged. In general, the effects observed upon oscillating or continuous HG were similar, with slight differences in few cases. Overall, under conditions of OM and HM, the changes persisted and were similar to those observed under OG and HG, despite the 7 days of growth in the presence of NG levels, confirming the occurrence of the metabolic memory phenomenon induced by both OG and HG. Overall, treatment with vildagliptin could revert these changes and the effect was more evident than upon sitagliptin, suggesting anti-oxidant and anti-apoptotic properties that can, at least in part, counteract the effects observed under HG-induced metabolic memory [5,16,37].

In a recent report, we assessed the antioxidant properties of another DDP-4 inhibitor, teneligliptin, in comparison with sitagliptin, on HUVECs grown under conditions of continuous NG, HG and HM [31]. Similarly to our results, teneligliptin was able to ameliorate the oxidative stress and apoptotic phenotype of HUVECs induced by continuous hyperglycemia, and to counteract the changes observed upon HM. Notably, the effects were dose-dependent and were more pronounced than upon sitagliptin [31].

The present study has some limitations. Indeed, all measurements of oxidative stress marker levels upon vildagliptin and sitagliptin were performed at only one time point (i.e., after $1 \mathrm{~h}$ of treatment), and thus no information is provided on the possible changes in ROS levels in HUVECs upon chronic administration of the drugs. Moreover, we showed that vildagliptin could ameliorate the oxidative status of HUVECs exposed to hyperglycemic conditions, but we did not investigate its capability to induce antioxidant enzymes, as this was beyond the focus of the present work. Therefore, additional studies are needed to define the protein expression profile for different markers.

Moreover, improvement on cellular redox state assessment is necessary to well-characterize the antioxidant capacity of vildagliptin. If our findings on the protective effects of vildagliptin on endothelial cells will be confirmed in vitro and in vivo, its use may be implemented as part of the therapeutic approach in diabetic patients, to prevent the onset of metabolic memory and, consequently, of vascular complications.

In conclusion, this study provides evidence supporting the protective role of vildagliptin in counteracting HG-induced oxidative stress and apoptosis in endothelial cells, and the metabolic memory induced by both oscillating and continuous HG conditions. 
Citation: Sala LL, Genovese S, Ceriello A (2017) Effect of Vildagliptin, Compared to Sitagliptin, on the Onset of Hyperglycemia-Induced Metabolic Memory in Human Umbilical Vein Endothelial Cells. Cardiovasc Pharm Open Access 6: 203. doi: 10.4172/2329-6607.1000203

A

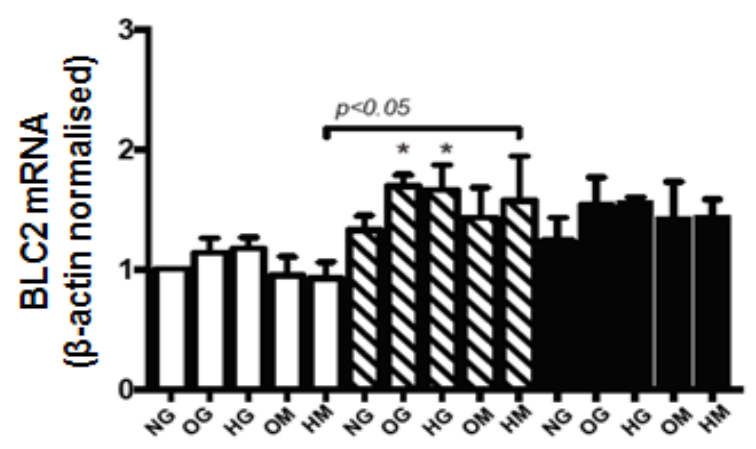

C



Caspase-3

$\beta$-actin

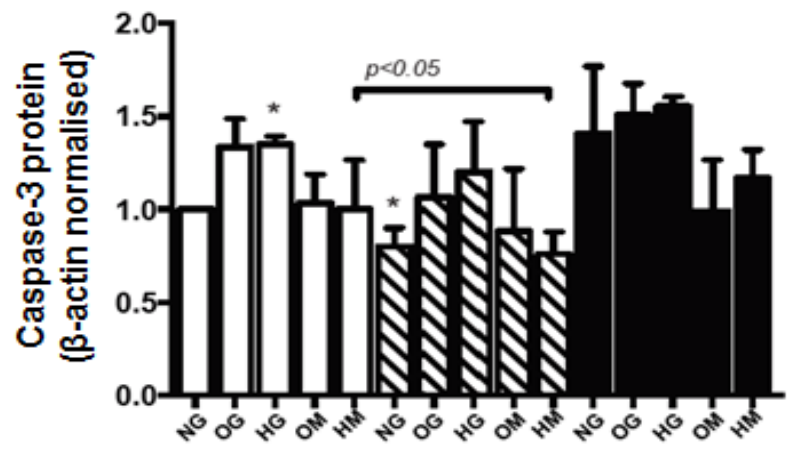

B



Figure 4: Regulation of apoptosis-related markers. Total RNA or proteins were extracted from HUVECs grown for 21 days under NG, OG, HG or OM-HM conditions and treated with vildagliptin $(5 \mathrm{nM})$ or sitagliptin $(5 \mathrm{nM})$ for $1 \mathrm{~h}$. The expression of BCL2 (A) and BAX (B) mRNA was quantified by qRT-PCR and normalized on $\beta$-actin. (C) $50 \mu \mathrm{g}$ of protein lysates were resolved on SDS-PAGE and the expression of caspase- 3 was normalized on the expression of $\beta$-actin. The experiment was performed

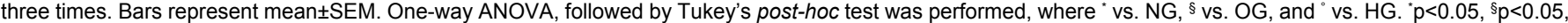
$\$ s p<0.01,{ }^{\circ} p<0.05,{ }^{\circ p}<<0.01$. NG: Normal glucose; OG: Oscillating high glucose; HG: Continuous high glucose; OM: OG memory; HM: HG memory.

\section{Acknowledgement}

Medical writing support and editorial assistance was provided by Clara Ricci, PhD (Primula Multimedia S.r.L., Pisa) and funded by Novartis (no grant number).

\section{Authorship Contributions}

LSL contributed to the acquisition, analysis and interpretation of data and manuscript writing. SG and AC contributed to analysis and interpretation of data and manuscript writing. All authors have read and approved the final manuscript.

\section{Funders}

Novartis funded medical writing and editorial assistance (no grant number).

\section{References}

1. Ceriello A (2003) New insights on oxidative stress and diabetic complications may lead to a "causal" antioxidant therapy. Diabetes Care 26: 1589-1596.

2. Brownlee M (2001) Biochemistry and molecular cell biology of diabetic complications. Nature 414: 813-820.

3. Ceriello A, Ihnat MA (2010) 'Glycaemic variability': a new therapeutic challenge in diabetes and the critical care setting. Diabet Med 27: 862-867.

4. Ceriello $A(2002)$ Role of hyperglycemia in nitrotyrosine postprandial generation. Diabetes Care 25: 1439-1443.

5. Schisano B (2011) Glucose oscillations, more than constant high glucose, induce p53 activation and a metabolic memory in human endothelial cells. Diabetologia 54: 1219-1226.

6. Gong $L$ (2011) Hyperglycemia induces apoptosis of pancreatic islet endothelia cells via reactive nitrogen species-mediated Jun $\mathrm{N}$-terminal kinase activation. Biochim Biophys Acta 1813: 1211-1219.

7. Laakso M (1999) Hyperglycemia as a risk factor for cardiovascular disease in type 2 diabetes. Prim Care 26: 829-839.

8. Ceriello A (2005) Postprandial hyperglycemia and diabetes complications: is it time to treat. Diabetes 54: 1-7.

9. Singleton JR (2003) Microvascular complications of impaired glucose tolerance Diabetes 52: 2867-2873.

10. Risso A (2001) Intermittent high glucose enhances apoptosis in human umbilical vein endothelial cells in culture. Am J Physiol Endocrinol Metab 281 e924-e930.

11. Quagliaro L (2003) Intermittent high glucose enhances apoptosis related to oxidative stress in human umbilical vein endothelial cells: the role of protein kinase $\mathrm{C}$ and $\mathrm{NAD}(\mathrm{P}) \mathrm{H}$-oxidase activation. Diabetes 52: 2795-2804.

12. Quagliaro $L$ (2005) Intermittent high glucose enhances ICAM-1, VCAM-1 and E-selectin expression in human umbilical vein endothelial cells in culture: the distinct role of protein kinase $\mathrm{C}$ and mitochondrial superoxide production. Atherosclerosis 183: 259-267.

13. Piconi $L$ (2006) Constant and intermittent high glucose enhances endothelia 
Citation: Sala LL, Genovese S, Ceriello A (2017) Effect of Vildagliptin, Compared to Sitagliptin, on the Onset of Hyperglycemia-Induced Metabolic Memory in Human Umbilical Vein Endothelial Cells. Cardiovasc Pharm Open Access 6: 203. doi: 10.4172/2329-6607.1000203

Page 6 of 6

cell apoptosis through mitochondrial superoxide overproduction. Diabetes Metab Res Rev 22: 198-203.

14. Takeuchi A (1995) Periodic high extracellular glucose enhances production of collagens III and IV by mesangial cells. Am J Physiol 268: F13-F19.

15. Jones SC (1999) Intermittent high glucose enhances cell growth and collagen synthesis in cultured human tubulointerstitial cells. Diabetologia 42: 1113-1119.

16. Ihnat MA (2007) Reactive oxygen species mediate a cellular 'memory' of high glucose stress signalling. Diabetologia 50: 1523-1531.

17. Roy S (1990) Overexpression of fibronectin induced by diabetes or high glucose: phenomenon with a memory. Proc Natl Acad Sci USA 87: 404-408.

18. Engerman RL, Kern TS (1987) Progression of incipient diabetic retinopathy during good glycemic control. Diabetes 36: 808-812.

19. Rajasekar P (2015) Epigenetic Changes in Endothelial Progenitors as a Possible Cellular Basis for Glycemic Memory in Diabetic Vascular Complications. J Diabetes Res.

20. Berezin A (2016) Metabolic memory phenomenon in diabetes mellitus: Achieving and perspectives. Diabetes Metab Syndr 10: S176-S183.

21. Ceriello A (2011) The possible protective role of glucagon-like peptide 1 on endothelium during the meal and evidence for an "endothelial resistance" to glucagon-like peptide 1 in diabetes. Diabetes Care 34: 697-702.

22. Mima A (2012) Protective effects of GLP-1 on glomerular endothelium and its inhibition by PKCbeta activation in diabetes. Diabetes 61: 2967-2979.

23. Duvnjak L, Blaslov K (2016) Dipeptidyl peptidase-4 inhibitors improve arteria stiffness, blood pressure, lipid profile and inflammation parameters in patients with type 2 diabetes mellitus. Diabetol Metab Syndr 8: 26.

24. Hamamoto $S$ (2013) Vildagliptin preserves the mass and function of pancreatic beta cells via the developmental regulation and suppression of oxidative and endoplasmic reticulum stress in a mouse model of diabetes. Diabetes Obes Metab 15: 153-163

25. Maeda S, Matsui T,Yamagishi S (2012) Vildagliptin inhibits oxidative stress and vascular damage in streptozotocin-induced diabetic rats. Int J Cardiol 158 171-173.
26. Chinda K (2013) Cardioprotective effect of dipeptidyl peptidase-4 inhibitor during ischemia-reperfusion injury. Int J Cardiol 167: 451-457.

27. Matsui T (2011) Vildagliptin blocks vascular injury in thoracic aorta of diabetic rats by suppressing advanced glycation end product-receptor axis. Pharmacol Res 63: 383-388.

28. Rizzo MR (2012) Reduction of oxidative stress and inflammation by blunting daily acute glucose fluctuations in patients with type 2 diabetes: role of dipeptidyl peptidase-IV inhibition. Diabetes Care 35: 2076-2082.

29. Ahren B (2011) Mechanisms of action of the dipeptidyl peptidase-4 inhibitor vildagliptin in humans. Diabetes Obes Metab 13: 775-783.

30. Davis JA (2010) Nature of action of Sitagliptin, the dipeptidyl peptidase-IV inhibitor in diabetic animals. Indian J Pharmacol 42: 229-233.

31. Pujadas G (2016) The dipeptidyl peptidase-4 (DPP-4) inhibitor teneligliptin functions as antioxidant on human endothelial cells exposed to chronic hyperglycemia and metabolic high-glucose memory. Endocrine.

32. Fisman EZ, Tenenbaum A (2015) Antidiabetic treatment with gliptins: focus on cardiovascular effects and outcomes. Cardiovasc Diabetol 14: 129.

33. Giacco F, Brownlee M (2010) Oxidative stress and diabetic complications. Circ Res 107: 1058-1070.

34. Nishikawa T (2003) Evaluation of urinary 8-hydroxydeoxy-guanosine as a novel biomarker of macrovascular complications in type 2 diabetes. Diabetes Care 26: 1507-1512.

35. Pujadas G (2016) The pivotal role of high glucose-induced overexpression of PKCbeta in the appearance of glucagon-like peptide-1 resistance in endothelial cells. Endocrine 54: 396-410.

36. Koya D, King GL (1998) Protein kinase C activation and the development of diabetic complications. Diabetes 47: 859-866.

37. Corgnali M (2008) Evaluation of gliclazide ability to attenuate the hyperglycaemic 'memory' induced by high glucose in isolated human endothelial cells. Diabetes Metab Res Rev 24: 301-309. 